\title{
A DTN-Based Multiple Access Fast Forward Serivce for the NASA Space Network
}

\author{
David J. Israel, Faith Davis, and Jane Marquart \\ Goddard Space Flight Center \\ Greenbelt, MD USA \\ e-mail: dave.israel@nasa.gov, faith.a.davis@nasa.gov, jane.k.marquart@nasa.gov
}

\begin{abstract}
The NASA Space Network provides a demand access return link service capable of providing users a space link "on demand". An equivalent service in the forward link direction is not possible due to Tracking and Data Relay Spacecraft (TDRS) constraints. A Disruption Tolerant Networking (DTN)-based Multiple Access Fast Forward (MAFF) service has been proposed to provide a forward link to a user as soon as possible. Previous concept studies have identified a basic architecture and implementation approach. This paper reviews the user scenarios and benefits of an MAFF service and proposes an implementation approach based on the use of DTN protocols.
\end{abstract} TDRS

Keywords- DTN; Fast Forward; scheduling; Space Network;

\section{INTRODUCTION}

The NASA Space Network (SN) or Tracking and Data Relay Satellite System (TDRSS) [1] provides communications and tracking services to many orbital and suborbital users, ranging from the International Space Station, Space Shuttle, and Hubble Space Telescope to Long Duration Balloons and Expendable Launch Vehicles. Using a constellation of Tracking and Data Relay Satellites (TDRS) in geosynchronous orbit, the SN offers full orbit coverage with bi-directional Radio Frequency (RF) links at $\mathrm{S}-\mathrm{Band}, \mathrm{Ku}-\mathrm{Band}$, and $\mathrm{Ka}-\mathrm{Band}$. Each TDRS has two Single Access (SA) antennas and a Multiple Access (MA) Phased Array Antenna system. All services are prescheduled by users with the exception of a Demand Access Service (DAS) which provides 1 to $150 \mathrm{kbps}$ S-Band return links to users "on demand" - without pre-scheduling [2].

The combination of phased array receive antennas, ground based beamforming, and spread spectrum exploited for DAS return links are not available in the forward link direction. Though the multiple access forward link system does use a phased array antenna approach, each TDRSS spacecraft only has the onboard electronics for up to two simultaneous forward links. The additional hardware for more simultaneous forward links, especially power amplifiers, will typically constrain relay spacecraft design. It is not possible for a forward link to always be available for a user on demand, because of this limitation. The best a user could get is a forward link as soon as possible. To emphasize this difference, the proposed new service is called Multiple Access Fast Forward (MAFF), instead of DAS Forward.

In order to provide MAFF service, user data processing at the missions operation center (MOC) and the Space Network Ground Station (SNGS) is required. The user data processing functions are an integral part of the MAFF service operation. An elegant and standard method of providing these processing capabilities can be achieved by utilizing the Disruption Tolerant Networking [6][7](DTN) protocols. Throughout this paper, references may be made to a "spacecraft", but it should be understood that this service may be used to support any user platform that may be in view of a TDRS.

\section{MAFF USER SCENARIOS AND BENEFITS}

The fundamental benefits of a MAFF service are the elimination of user MA Forward Link scheduling operations and the reduction in wait time or latency between data generation and transmission.

MA Forward Link service, like all other TDRSS services except DAS Return, requires scheduling. Schedules are typically finalized one week before an event, although, a user could identify and request TDRSS Unused Time, (TUT) within the active schedule window. The involvement of the user operations team is required in order to perform all of the identification and requests for event times. Identifying available time on relatively short notice (within a week) is an operator intensive activity, due to the fact that it requires TUT time for a particular TDRS that is in view of the user. For this reason, it is typical for a user to request periodic forward link events during the forecast scheduling period up to three weeks in advance to ensure that enough forward link time will be available "just in case". Any data that a MOC has ready to transmit to a spacecraft must wait until the next scheduled forward link event. To accommodate data with latency requirements, forward link events must be scheduled at intervals small enough to guarantee the maximum latency requirements. The combination of these factors not only increase the load on MOC scheduling operations, but they also lead to the underutilization of forward link time when a scheduled event occurs without any required forward link data to transmit. 


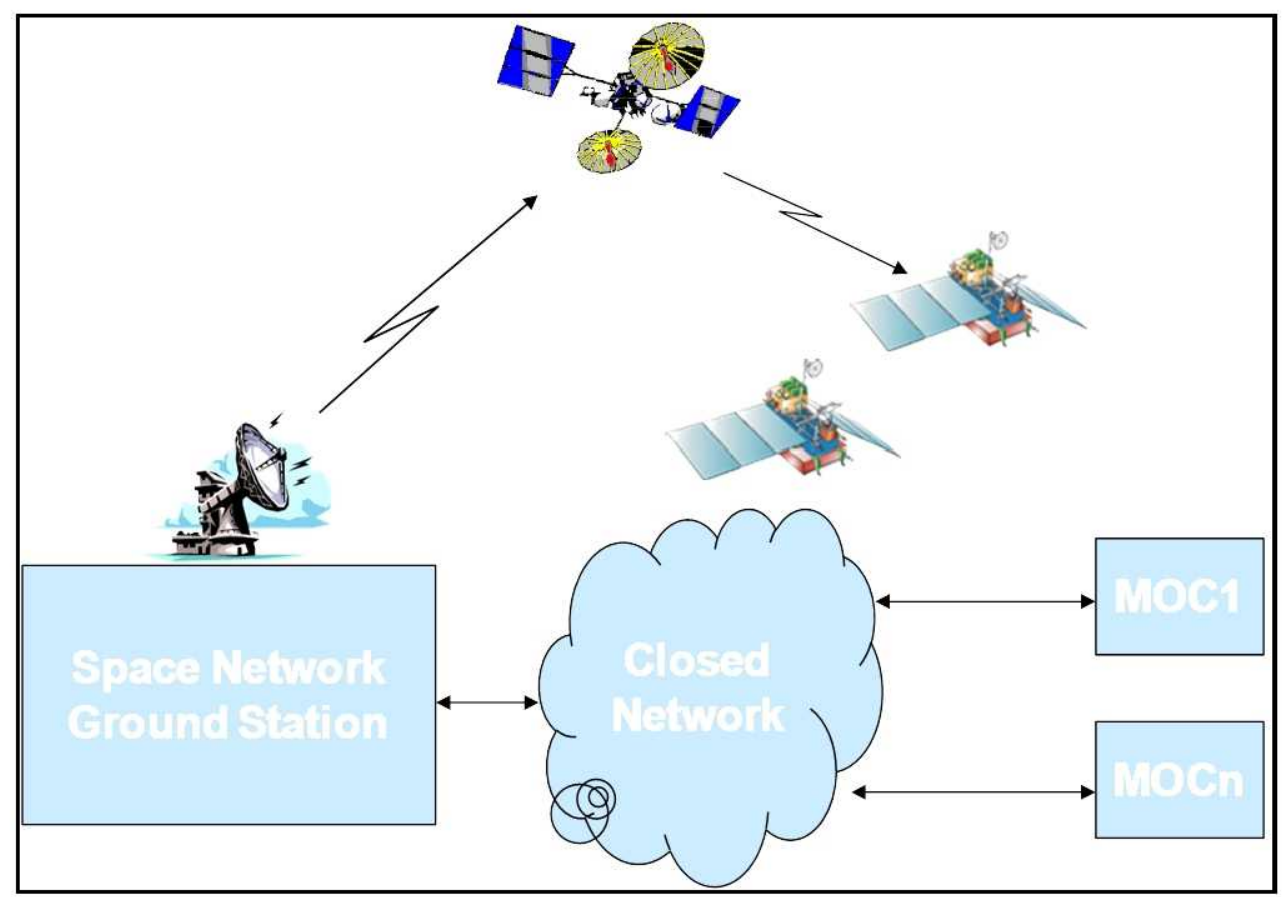

Figure 1-Multiple Access Forward Link Service

As seen in Figure 1, during an MA Forward Link Service event, forward link data from a MOC is sent to a SNGS, where it is modulated onto the Ku-Band Space-to-Ground Link (SGL) uplink to be relayed to the user spacecraft. Using an electronically steered phased array, the MA Forward Link signal is pointed in the direction of the user to a user spacecraft.

The proposed MAFF service would appear the same as a scheduled service - the power level, modulation, data rate, and coding would be identical. The difference would be that a MAFF service would not be pre-scheduled. In the pre-scheduled case, the MOC holds all data until the event begins. In the MAFF case, the MOC sends the data to the SNGS at any time and, as soon as possible, the MAFF system provides a MA Forward Link event to forward the data to the user spacecraft. This data driven approach makes the MAFF service ideal for the transmission of short messages such as science event notifications, acknowledgements of DAS return link only trans/missions, or retransmission requests.

A user platform may be part of a larger Earth Science sensorweb [3] or other collaborative science activity, such as the Gamma-ray burst Coordinates Network [4]. These science driven events cannot be scheduled in advance. The timeliness of a science opportunity notification to a user platform directly affects the ability of a platform to participate in a collaborative observation. If MAFF service were available, the MOC or other notification source would minimize latency by forwarding the event notification through the network to the SNGS, triggering the transmission to the user platform.

Some other operations scenarios couple MAFF with the use of the existing DAS service. DAS can provide either continuous return link only service or on demand return link only service. Currently, missions that are using continuous DAS service for streaming telemetry or the transfer of data files must schedule a forward link service to either acknowledge the successful reception of data, request retransmissions, or send commands. The amount of forward link time required for acknowledgement and retransmission requests is not completely deterministic, since it depends upon the return link performance (i.e., the more errors in the return link, the more retransmission requests). To account for the worst case scenarios, a user will currently schedule the maximum forward link time that may be necessary. This leads to scheduled MA Forward Link time that may be sending "No-Op" commands while another user waits for its turn. This is an inefficient use of the $\mathrm{SN}$ asset and creates unnecessary latency for the waiting user. Providing timelier retransmission requests or acknowledgements of data reception will also allow for more efficient storage management onboard the spacecraft system.

For the case when a DAS user employed DAS for on demand transmissions only, MAFF provides an unscheduled means for an acknowledgement of the DAS transmission. Whether the DAS transmission was a science event notification or an emergency "911" message, the ability for a more rapid acknowledgement from the MOC would enable more efficient spacecraft operations, since the 


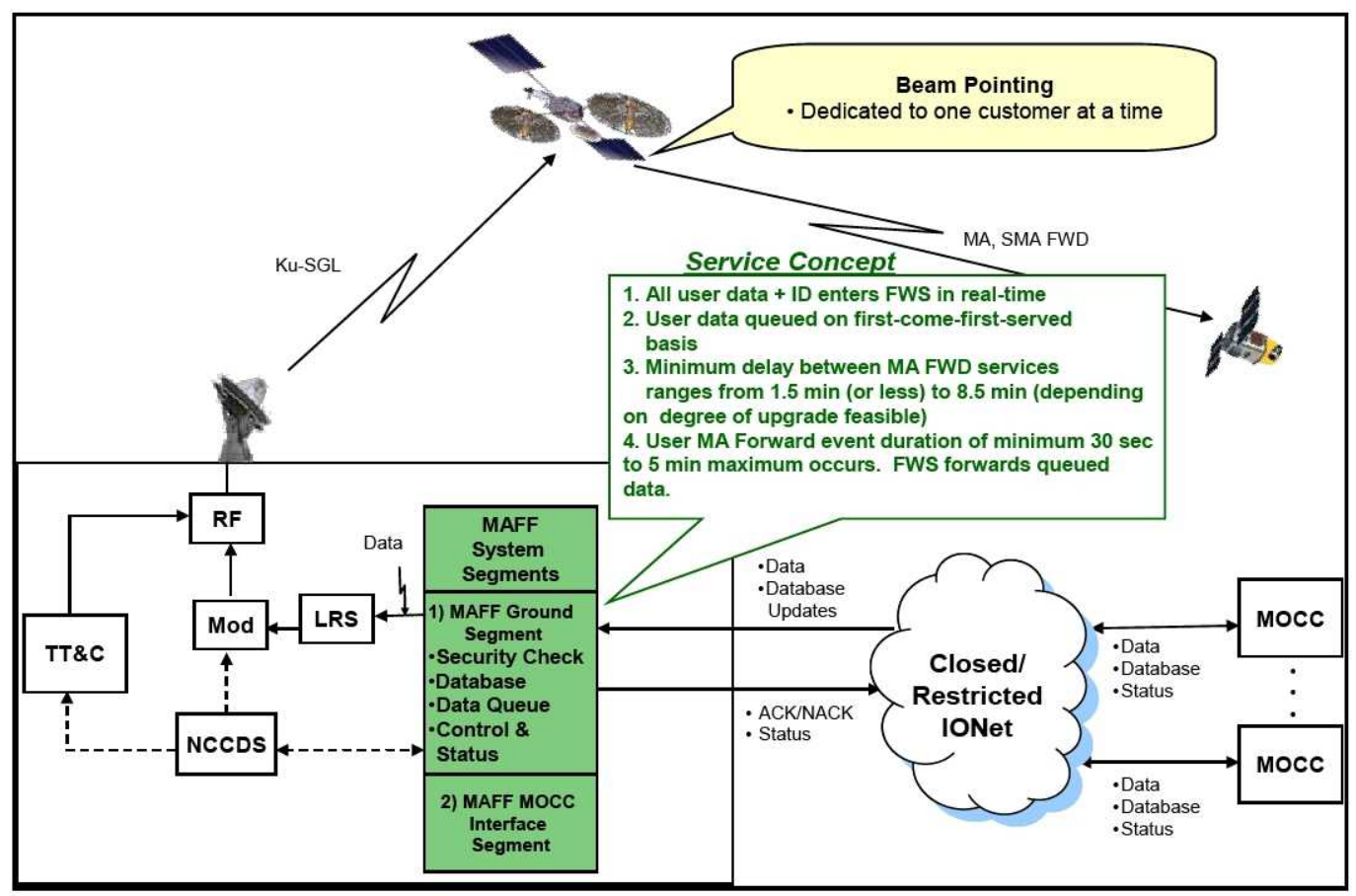

Figure 2 - 2006 MAFF Reference Architecture

spacecraft could proceed with the knowledge that the MOC received the message.

\section{MAFF IMPLEMENTATION CONCEPT}

The MAFF Project had successfully completed a Systems Requirement Review by the time the project was put on hold in early 2006. A reference architecture from that time period is illustrated in Figure 2 [5]. The MAFF Ops Concept included a Fast Forward Workstation (FFWS) that would receive user data intended for an MAFF link and serve as a proxy to request MA Forward time on behalf of a user. The FFWS also had to perform many required functions to handle the user data, including:

- Identification of the user, based on the data source

- Verification of the data source (Authentication)

- Storing and queuing of the data per user

- Forwarding the correct user data to the MA Forward communications system at the correct time

- Provision of status and statistics of data transfer back to the MOC.

At the time, a method for performing these functions was not identified. It appeared as if customized protocols would be necessary. In the years since, it has become apparent that the developing DTN protocols will provide the ability to perform these functions in a standardized manner that will not only meet the MAFF requirements, but will also tie the MAF service into a larger Solar System Internetwork (SSI) [8].

The following discussion of a DTN-Based MAFF Implementation Operations Concept will be at a high level. The details concerning how the DTN protocols work and the specific protocol details are beyond the scope of this paper. For the purposes of this paper, the reader should understand the following attributes of DTN:

- DTN provides network layer functionality (addressing, multi-hop routing, etc.)

- DTN is designed for disrupted links, including those disrupted due to lack of link availability

- DTN provides standardized protocols for store and forward data communications, including security, and reporting

- A DTN network includes Bundle Agents which perform the store and forward services at each hop within the network.

The DTN-Based MAFF Reference Architecture is illustrated in Figure 3. An application located at the MOC (or anywhere the mission allows to source data for the forward link) creates DTN bundles with a destination End 


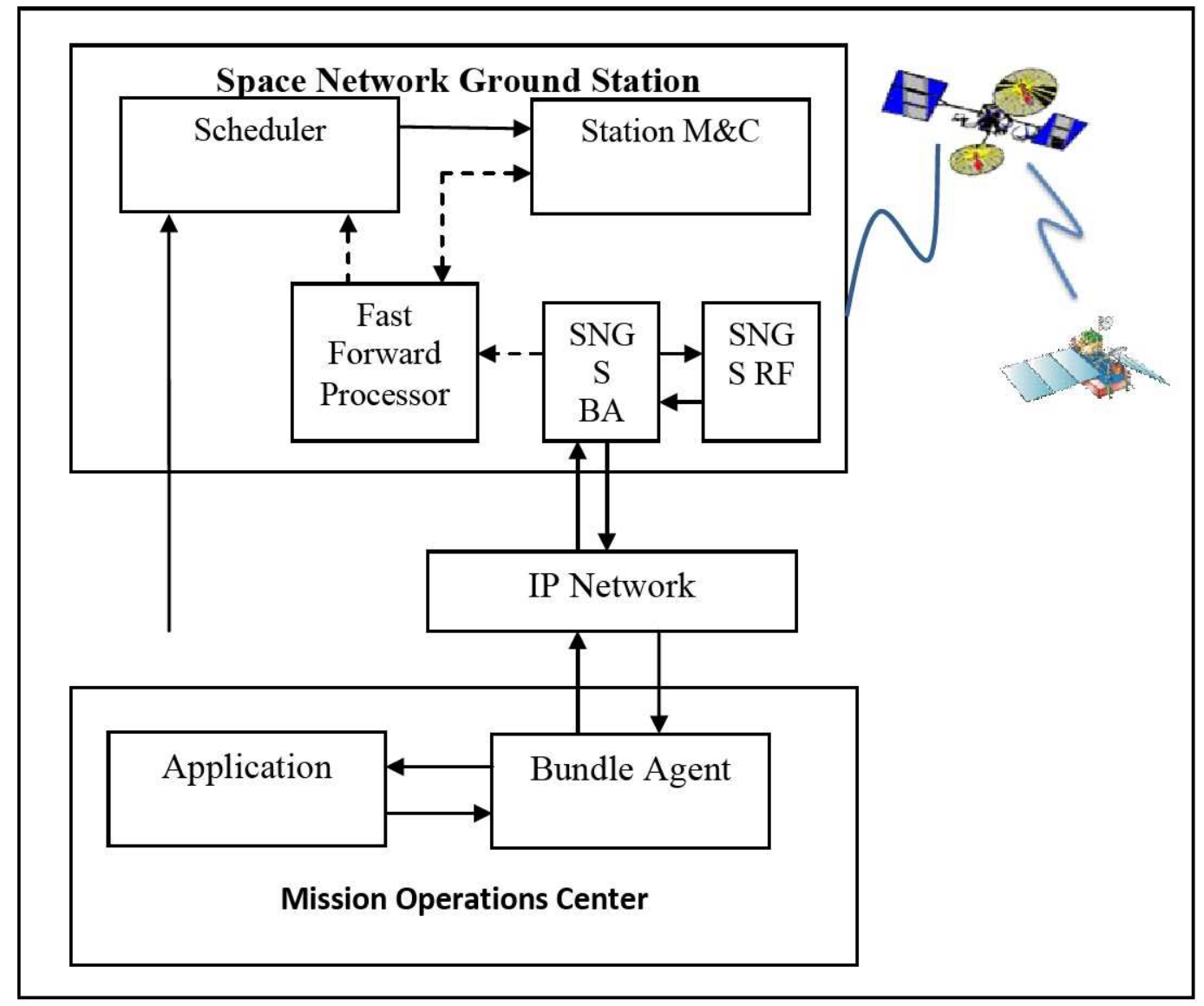

Figure 3 - DTN-Based MAFF Implementation Reference Architecture

Point Identifier (EID) assigned to the user spacecraft. A Bundle Agent (BA) located at the MOC will be able to store the bundles and forward them at the appropriate time based on a DTN routing protocol or static routing. If the route selected is the MAFF service, then the DTN bundles will be forwarded over an IP network to a Space Network Ground Station Bundle Agent (SNGS BA). The DTN Bundle Protocol (BP) and Bundle Security Protocol (BSP) will perform the addressing and authentication required for this transfer of data and the use of TCP/IP between the MOC and SNGS will provide reliability and routing across the terrestrial network.

When the bundles sent by the MOC BA are received at the SNGS, the SNGS BA will queue and store the bundles in a manner defined by the DTN protocols. Since DTN has developed or is already developing methods for the prioritization of data, deletion of data after a certain timeout, and the optional notification of data transmission, the SNGS BA will be able to address a majority of the identified MAFF requirements without the development of new protocols.

The non-DTN applications still required at the SNGS involve the scheduling of the MAFF service. A Fast Forward Processor (FFP), which could be a process within the same system as the SNGS BA, would perform these additional functions. The FFP would monitor the SNGS BA to determine which users have bundles waiting for transmission. When a user is identified, the FFP would process the user and TDRSS orbital information and combine it with knowledge of available MA Forward Link time to generate a schedule request for MA Forward Link time on a specific TDRS. This approach allows for the support of scheduled MA Forward Link and MAFF service on the same TDRS. When a user's MAFF service time starts, the FFP or possibly the SNGS Monitor and Control system notifies the SNGS BA that a link is available and the SNGS BA sends the data out over a connection to the proper MA Forward Link equipment connected to the Space-to-Ground Link (SGL) uplink.

The same SNGS BA could also be able to provide service for any return link bundles received via DAS or other $\mathrm{SN}$ return service, as well as other scheduled forward link services. This would enable full DTN capability across the SN links. The MAFF path from the BA would then be one of multiple bundle routing options.

This reference architecture may be used even in cases when the users do not desire DTN bundles to be transmitted across the spacelink. For these cases, the DTN protocols are only used terrestrially to facilitate all of the store and forward addressing, routing, authentication, and Quality of Service services. An additional function is required at the output of the SNGS BA which would strip the contents out of a DTN bundle before forwarding the data on for modulation onto the Forward Link. The user MOC would 
also be required to perform the original bundling of the data to forward it to the MAFF system.

\section{NEXT STEPS}

The next steps in the development of DTN-Based SN MAFF services fall into two main categories. The first category is the further development of the ops concept as it relates to use of $\mathrm{SN}$ resources and integration into the larger SN architecture. The second category is the test and demonstration of the DTN protocols to verify that they will be able to perform the required MAFF functions as described in this paper.

Ops Concepts: Since there are a limited number of MA Forward Link resources on orbit, an analysis of the ability to support both scheduled and MAFF links needs to be completed. Some scheduled MA Forward Links will likely be required, since, among other reasons, it is the only way to guarantee a simultaneous forward and return link to support coherent operations. If the forward links are mostly scheduled, then the response time for "as soon as possible" may not meet the user needs. There is also ongoing study into the provision of a beacon service using the MA Forward Link capability. This beacon service could provide a signal and data for improved navigation, space weather alerts, and network status. The beacon service may also allow for the multiplexing of user data within the broadcast data stream. The space allocated for user data could be filled with data provided from a MAFF service, allowing MAFF and a beacon service to share the same link. The impact on the MAFF service would be a reduction in the effective user data rate, a likely increase in latency, and the requirement to receive a separate beacon signal.

Test and Demonstration: The test and demonstration of the end-to-end MAFF service will be performed at the NASA Goddard Space Flight Center Communications, Standards, and Technology Laboratory (CSTL) [9]. The RF systems in the CSTL are implemented using software defined radio (SDR) technology and NASA engineering model transponders. Various link layer, modulation and coding schemes have been developed and tested, including the Tracking and Data Relay Satellite System (TDRSS) spread spectrum waveforms. Flight components include mission flight software and hardware, onboard LANs, and spacequalified routers. The DTN protocols are being tested for flight worthiness. The ground support system includes both commercial and government developed command/telemetry equipment. As such, this lab has the ability to emulate each element in the end-to-end communications scenario (i.e., user spacecraft, TDRSS, SNGS, and MOC), incorporate live links with the SN, and exercise DTN in the proposed MAFF service. Testing of the MAFF concept will include the configuration of the CSTL to match the architecture described here and simulating multiple MAFF scenarios, in order to determine if the DTN protocols can meet the operational requirements.

\section{SUMMARY}

The idea of eliminating scheduling and achieving more efficient use of the forward link through the MAFF service has been under consideration for over five years. Until the arrival of the DTN protocols, its implementation seemed to require a lot of custom software. The proposed path forward, described in this paper, could enable a clean, standardized, way of fulfilling the data handling requirement for a MAFF service. Further development of the operations concept, testing, and demonstration of the end-to-end MAFF service in the CSTL are the next steps in developing this concept. Data gathered from this process will be used to determine the feasibility of the proposed MAFF service for the SN.

\section{REFERENCES}

[1] Space Communications and Navigation TDRSS Website https://www.spacecomm.nasa.gov/spacecomm/progr ams/tdrss

[2] T. Gitlin, W. Kearns, and W. Horne, "The NASA Space Network Demand Access System (DAS)," SpaceOps 2002, Houston, 2002.

[3] K. Moe; S. Smith, G. Prescott, R. Sherwood, "Sensor Web Technologies for NASA Earth Science". Proceedings of 2008 IEEE Aerospace Conference, pp. 1-7, 2008.

[4] Gamma-ray bursts Coordinates Network Website. http://gcn.gsfc.nasa.gov/

[5] Draft Space Network Multiple Access Fast Forward (MAFF) Operations Concept Document (OCD), 452-OCD-MAFF, January 2006.

[6] Disruption Tolerant Networking Research Group Website http://www.dtnrg.org/

[7] Keith L. Scott, "Space Internetworking with IP and DTN," SpaceOps 2008, Heidelberg, 2008.

[8] "Operations Concept for a Solar System Internetwork (SSI)", Interagency Operations Advisory Group Report, October 2010.

[9] Israel, D.J.; Marquart, J.K.; Thompson, W.L.; , "The GSFC Communications, Standards, and Technology Laboratory (CSTL)," Aerospace Conference, 2008 IEEE, vol., no., pp.1$7, \quad 1-8 \quad$ March 2008 